\title{
Taper designs for semiconductor filled fibres
}

Neil G. R. Broderick and Anna C. Peacock

Optoelectronics Research Centre, University of Southampton, Southampton, SO17 1BJ, UK.

Phone: +44 (0)23 80597696, Fax: +44 (0)23 80593142, email: ngb@orc.soton.ac.uk

Recently there has been much interest in designing optical tapers for nonlinear pulse manipulation in both standard[1] and semiconductor filled fibres[2]. Such tapers can be used to overcome the large linear losses in semiconductor filled fibres while allowing parabolic pulse generation or soliton propagation for example. However once the taper profile has been chosen to overcome the linear loss problem in semiconductor filled fibres the problem of two photon absorption (TPA) and associated free carrier effects remain. In this work we show that it is possible to use a genetic algorithm to design tapers that alleviate both linear and nonlinear losses and hence should allow high quality pulse propagation over several centimetres of semiconductor filled fibres.

In a tapered semiconductor filled optical fibre the pulse envelope obeys the modified nonlinear Schrödinger equation with varying coefficients[2]:

$$
i \frac{\partial \psi}{\partial z}-\frac{\beta_{2}(z)}{2} \frac{\partial^{2} \psi}{\partial t^{2}}+i \frac{\beta_{3}(z)}{6} \frac{\partial^{3} \psi}{\partial t^{3}}+i\left(\sigma_{f}+\frac{\alpha}{2}\right) \psi+\gamma(z)|\psi|^{2} \psi=0
$$

where the normalised pulse envelope is given by $\psi$ while the effect of tapering is included in the position dependent coefficients $\beta_{2}, \beta_{3}$ and $\gamma$. The effects of TPA are included by making $\gamma$ complex, whilst the free carrier effects (absorption and dispersion) are described by $\sigma_{f}$. Full details of this along with the associated evolution equation for the free carrier density are given in Ref. [2]. For the semiconductor filled fibres treated here, the analytic solutions for the modes of a step index fibre[3] can be used to find values for the different parameters once the core radius $r$ is given (see Fig. 1a). Thus a taper is completely described by the function $r(z)$ which can take values between $200 \mathrm{~nm}$ and $1.5 \mu \mathrm{m}$ for the tapers under consideration.

In contrast to previous work[1] where a genetic algorithm was used only to optimise $\beta_{2}$ here we used a genetic algorithm to optimise the core radius. This ensures that at all times the taper is physically realisable and that the correct values of higher order dispersion are used. In addition we allowed the genetic algorithm to determine the input pulse width and amplitude. The genetic algorithm was implemented using an OpenMPI implementation on a linux cluster and a typical run would use 400 processors and take about 5 hours.

In order to compare our results with previous results we looked at two examples. The first being parabolic pulse generation in a $2 \mathrm{~mm}$ fibre taper and the second being soliton propagation through $2 \mathrm{~cm}$ of fibre using the same parameters as in Ref. [2]. Here the effects of TPA are fairly small and so the taper designs are similar to those found previously however in each case the misfit parameter that describes the output pulse mismatch has been reduced by at least an order of magnitude compared to previous results (Ref. [2]).

In conclusion we have shown that it is possible to design tapers using a genetic algorithm to overcome the effects of both extremely high linear loss and TPA. Using this algorithm in the future will result in improved propagation characteristics in semiconductor filled fibres and thus increase their usefulness as nonlinear waveguides.

[1] N. G. R. Broderick, "Method for pulse transformations using dispersion varying optical fibre tapers" Opt. Ex. 18 pp 24060 (2010).

[2] A. C. Peacock and N. Healy, "Parabolic pulse generation in tapered silicon fibers", Opt. Lett. 35 (11) (2010).

[3] A. W. Snyder and J. D. Love, "Optical Waveguide Theory", Kluwer Academic Publishers, 2000.
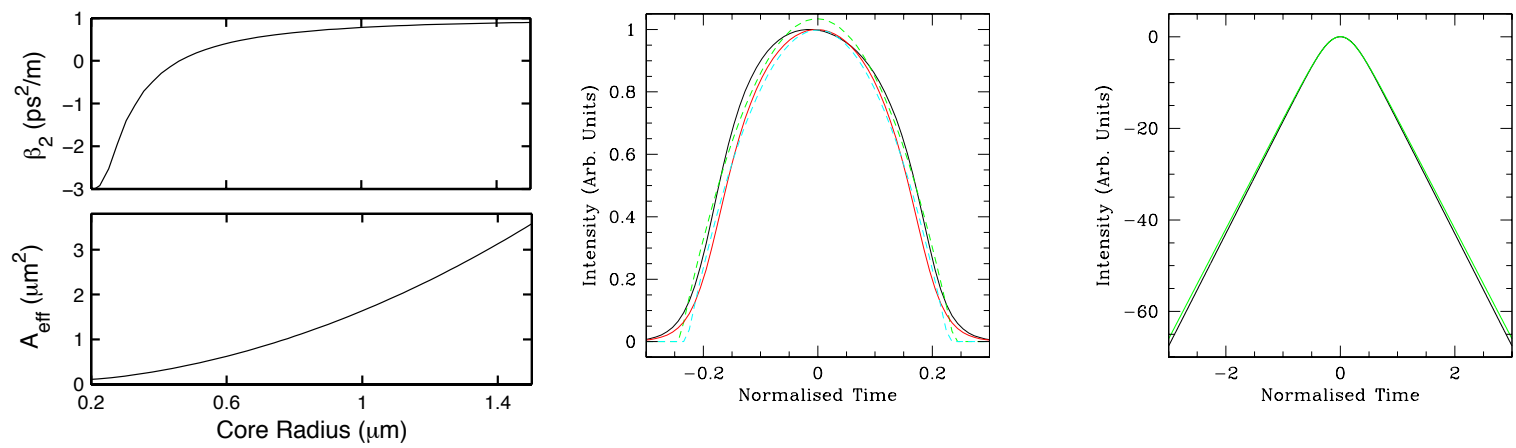

FIG. 1. (a) Effect of tapering on fibre parameters. (b) Parabolic pulse generating with (red line) and without (black line) free carrier effects, (c) Soliton profile after $2 \mathrm{~cm}$ of propagation. Green lines show the ideal shapes in (b) and (c). 